\title{
Anti-Aging Medicine: Molecular Basis for Endothelial Cell-Targeted Strategies - A Mini-Review
}

\author{
Giovanni G. Camici ${ }^{\mathrm{a}} \mathrm{Yi} \mathrm{Shi}^{\mathrm{a}} \quad$ Francesco Cosentino $^{\mathrm{a}-\mathrm{c}}$ Pietro Francia ${ }^{\mathrm{c}}$ \\ Thomas F. Lüscher ${ }^{a, b}$ \\ ${ }^{a}$ Cardiovascular Research, Institute of Physiology, and Center for Integrative Human Physiology (ZIHP), University \\ of Zürich, and b Department of Cardiology, Cardiovascular Center, University Hospital, Zürich, Switzerland; \\ 'Cardiology, 2nd Faculty of Medicine, Sapienza University of Rome, Sant'Andrea Hospital, Rome, Italy
}

\section{Key Words}

Calorie restriction - Free radical-induced damage $\cdot$

Reactive oxygen species

\begin{abstract}
Due to improvements in lifestyle and healthcare, the proportion of aged people is rising steadily, especially in developed countries. With aging, some physiological functions are altered and resemble those occurring in disease conditions such as hypertension, chronic coronary disease and diabetes. Thus, there is the urge to better understand molecular and cellular mechanisms underlying aging and aging-related diseases. In rodents and possibly primates, calorie restriction is an effective approach to extend lifespan by reducing free radical-induced damage. Increased production of oxygen-derived free radicals plays an important role in the process of aging. Reactive oxygen species are generated by different intracellular molecular pathways principally located in the cytoplasm and in the mitochondria. The mitochondrial protein $\mathrm{p} 66^{\mathrm{Shc}}$ is considered a longevity assurance gene since its genetic deletion extends the lifespan of rodents and displays protective effects in several models of cardiovascu-
\end{abstract}

lar disease. Silent mating type information regulation 2 homolog 1 Saccharomyces cerevisiae (SIRT1) is a nicotinamide adenine dinucleotide (NAD+)-dependent histone deacetylase that may also be involved in aging and diseases. SIRT1 also deacetylates a number of nonhistone target proteins, including p53, endothelial nitric oxide synthase and forkhead box protein. This review focuses on the latest scientific advances in understanding aging as well as delineates the possible therapeutic implications of $\mathrm{p} 66^{\text {Shc }}$ and SIRT1 in this process.

Copyright $\odot 2010$ S. Karger AG, Basel

Aging is an inevitable part of life. Human life expectancy has risen impressively over the last centuries due to significant improvements in hygiene and health care. Consequently, the proportion of aged people is steadily increasing. With aging, some physiological functions of organs are altered and resemble those occurring in disease conditions such as hypertension, chronic coronary disease and diabetes [1]. Moreover, cardiovascular disease correlates with aging even in the absence of established risk factors, suggesting that aging per se is an in-

\section{KARGER}

Fax +41613061234

E-Mail karger@karger.ch

www.karger.com
(C) 2010 S. Karger AG, Basel

0304-324X/11/0572-0101\$38.00/0

Accessible online at:

www.karger.com/ger
Thomas F. Lüscher, MD, FRCP

Department of Cardiology, Cardiovascular Center, University Hospital Rämistrasse 100

CH-8091 Zürich (Switzerland)

Tel. +41 44255 2121, Fax +41 44255 4251, E-Mail cardiotfl@gmx.ch 
dependent risk factor for the development of cardiovascular disorders. Therefore, aging and aging-related diseases currently represent an important research topic.

Calorie restriction is a widely used protocol for life span studies in experimental animals (rodents and primates) [2]. It was first reported in 1935 that white rats fed with a low-calorie diet have an extended life span, lower body weight, lower heart weight and smaller bone size [3]. However, there are still unanswered questions concerning the global effects of calorie restriction. Indeed, as spontaneous cancer develops with aging in rodents, the real role of calorie restriction in animals is often confused with a reduction in the incidence of cancer, extension of life span, or both. Moreover, it is not fully understood whether the beneficial effects of calorie restriction also extend to humans; however, if they do, the underlying mechanisms have yet to be elucidated.

Interestingly, in a 20-year longitudinal adult-onset calorie restriction study in rhesus monkeys, $30 \%$ calorie restriction significantly reduces aging-related deaths [4]. Monkeys in the calorie-restricted group conserved glucose homeostasis, indicating a beneficial effect of calorie restriction on diabetes. The incidence of cancer increases with aging in rhesus monkeys, confirming the spontaneous development of cancer in aged rodents and indicating that cancer is an aging-related disease as well. However, monkeys under calorie restriction have a lower incidence of cancer compared with control animals. Calorie restriction also reduces the incidence of cardiovascular diseases in the same group. In addition, aged monkeys have been shown to develop brain atrophy in the bilateral frontal and temporal cortex in both control and diet-treated groups, suggesting a pathophysiological change observed with aging which is independent of calorie intake. However, monkeys on a low-calorie diet maintain grey matter volume in subcortical regions [4], supporting the finding that calorie restriction preserves locomotory activity in aged monkeys [5]. These results underscore the beneficial effects of calorie restriction on aging and aging-related diseases in primates.

In humans, Okinawan populations consuming 20\% less calories compared to the average caloric consumption of the Japanese population display reduced mortality in elderly people and also decreased death rates of $30-40 \%$ due to lower incidences of cancer, cerebrovascular and heart diseases. Furthermore, Okinawans who switch to a Western diet are shown to have similar mortality and morbidity as people from Western countries. These data confirm the beneficial effects of calorie restriction in humans, although it cannot be excluded that a normal diet may adversely affect longevity independently of its caloric content. Strikingly, the data from members of the Calorie Restriction Society show consistent and positive effects of calorie restriction in adults. Compared with subjects on normal Western diets, subjects on a low-calorie diet have less risk factors for atherosclerosis, including reduced body mass index and blood pressure (both systolic and diastolic), lower levels of glucose, insulin, tumor necrosis factor- $\alpha$ and C-reactive protein, decreased levels of triglycerides and lowdensity lipoprotein (LDL)-cholesterol, and higher levels of high-density lipoprotein (HDL)-cholesterol. These beneficial effects are shown after one year of caloric restriction and improve further after six years of restriction [6]. Likewise, a Mediterranean diet characterized by large quantities of olive oil also has protective effects on cardiovascular risk factors [7].

\section{The Free Radical Theory of Aging}

The free radical theory of aging was first proposed by Denham Harman in the 1950s and states that production of reactive oxygen species (ROS), occurring as part of normal cellular metabolism, increases with aging and further contributes to the process of aging. Hydroxyl radicals and hydrogen peroxide $\left(\mathrm{H}_{2} \mathrm{O}_{2}\right)$ are considered to play an important role in the process of aging [8]. However, the exact source of increased ROS observed in aging is still unclear. Mitochondria are one of main sources of intracellular superoxide anion radical $\left(\mathrm{O}_{2}^{-}\right)$and $\mathrm{H}_{2} \mathrm{O}_{2}$, thus they are thought to experience heavy oxidative damage with aging. The life-time exposure of mitochondria to ROS damage in turn enhances ROS production, which ultimately and negatively affects life span. Enhanced production of ROS in aging is also associated with upregulated expression of inducible nitric oxide synthase, and nicotinamide adenine dinucleotide phosphate-oxidase and/or downregulation of antioxidants, such as extracellular superoxide dismutase, glutathione peroxide and decreased catalase activity [9].

The increased production of ROS observed with aging contributes to oxidizing lipids, consequently modifying membrane lipid composition and reducing its fluidity. The mitochondrial membrane is particularly susceptible to peroxidation because of its high composition of unsaturated lipids. Aging and aging-related ROS production increase mitochondrial membrane rigidity and further reduce efficiency of mitochondrial oxidative phosphorylation, resulting in cell apoptosis [10]. Calorie 
restriction and a Mediterranean-style diet maintain normal membrane composition by reducing the index of unsaturation/saturation of lipids, thus blunting ROS-mediated damage of cell membranes [11].

Protein carbonyl, a protein oxidation marker, has been shown to increase with aging in the mouse brain and skeletal muscle mitochondria [12]. However, data concerning the effect of calorie restriction on protein carbonyl content are not consistent. Indeed, mitochondrial carbonyl concentration was not significantly changed in mice under calorie restriction [13].

Aging-related ROS also damage mitochondrial DNA (mtDNA) as confirmed by the elevated frequency of deletion occurring in mtDNA. An increased level of deletion frequency of mtDNA is observed in the brain, liver, heart and skeletal muscle of old rats and a calorie restriction diet decreases the higher level of deletion frequency in liver of aged mice [14]. Hydroxytyrosol, an important polyphenolic compound in olive oil, stimulates mitochondrial transcription factor $\mathrm{A}$ and increases the amount of mitochondria inside of cells, therefore maintaining mitochondrial functions [15].

\section{The Effect of Calorie Restriction on ROS Generation}

It is generally believed that the effect of calorie restriction on life span is due to a reduced metabolic rate and a decreased mitochondrial oxygen consumption. Nevertheless, pigeons have a comparable metabolic rate and body mass to rats, but have an eightfold longer life span. More importantly, the rate of $\mathrm{H}_{2} \mathrm{O}_{2}$ generation in mitochondria is lower in long-lived rather than short-lived animals. Thus, it is logical to correlate the rate of aging and maximum longevity to the rate of mitochondrial ROS generation [16].

In mitochondria, aging-related ROS production is mainly due to reduced activity of mitochondrial electron chain complexes [17]. The activity of the four electron transport systems is decreased with aging in human as well as in rodent skeletal muscles. Calorie restriction maintains the activity of the four electron complexes, thus reducing ROS production in mitochondria [17]. In addition, calorie restriction has a direct effect on antioxidant enzymes. In rat liver, calorie restriction is shown to restore catalase and glutathione peroxidase activity, as well as upregulating the expression of $\mathrm{Cu}-\mathrm{Zn}$ superoxide dismutase [18]. It is also reported that calorie restriction prevents the decrease of glutathione and glutathione transferase activity in rat skeletal muscle [19].

Aging, p66 ${ }^{\text {Shc }}$ and SIRT1
On the whole, calorie restriction reduces the generation of free radicals due to attenuation of mitochondrial damage and upregulation of defense enzymes. Strict control of calorie intake is, however, an unpleasant and tough experience. Severe long-term restriction could induce malnutrition, especially in aged people. Meanwhile, in large prospective clinical trials, neither the separate nor the combined antioxidant treatment with vitamins $\mathrm{C}$ and E significantly reduced the incidence of major adverse cardiovascular events. This could be explained by several factors: (1) natural antioxidants are commonly found in normal human daily diet and, thus, supplements may have no additional effects; (2) additional antioxidant treatment may depress endogenous antioxidant defense systems, thus preventing the expected positive effects of antioxidant therapy; and (3) the intracellular pathway may be sequestered in organelles (for instance mitochondria) and antioxidants are not reachable for the compound. Therefore, there is a call for new therapeutics other than dieting and antioxidant treatment to prolong life span and to improve life quality.

\section{Mammalian Longevity Assurance Gene Sirtuins}

Silent mating type information regulation 2 homolog (Sir2) family proteins, sirtuins, are considered to be crucial regulators of life span extension in the setting of calorie restriction. Calorie restriction activates Sir2 protein in yeast Saccharomyces cerevisiae and flies. SIRT1, the mammalian Sir2, is consistently upregulated in mice under calorie restriction. SIRT1 protein is a nicotinamide adenine dinucleotide (NAD+)-dependent histone deacetylase, which also deacetylates a number of nonhistone target protein, including tumor suppressor $\mathrm{p} 53$ protein, members of the forkhead transcription factor family (FoxO), stress response proteins Ku70 and nuclear factor $\kappa$-light-chain-enhancer of activated B cells, and metabolic regulator $\alpha$-subunit of the G protein (PCG-1 $\alpha$ ) $[20,21]$. Starvation enhances the expression of SIRT1 protein in mouse liver and in human peripheral blood mononuclear cells, which returns to a normal level upon feeding [22], indicating that SIRT1 is sensitive to nutrition and metabolic changes. The upregulated SIRT1 protein deacetylates PGC-1 $\alpha$, a transcriptional coactivator that controls energy state in a NAD+-dependent manner in hepatic cells from both vivo and vitro experiments, suggesting a regulatory role of SIRT1 in mitochondrial biogenesis and energy metabolism [22]. Thus, SIRT1 is an important mediator of the beneficial effects observed 
with calorie restriction, and is probably involved in life span control.

The presence of SIRT1 protein is also observed in pancreatic $\beta$-cells [23]. In cell culture experiments, inhibition of SIRT1 reduces insulin secretion, suggesting a role of SIRT1 in insulin secretion [23]. In the aorta of streptozotocin-induced type I diabetes mice, the expression of SIRT1 protein is reduced while acetylated p53 and p21 proteins are upregulated [24]. Resveratrol, a small-molecule activator of SIRT1, deacetylates p53 and p21 in the aorta, thereby improving endothelial dysfunction by downregulating intercellular adhesion molecule-1 expression and inhibiting leukocyte rolling, which suggests a protective role for SIRT1 in diabetes-induced vascular dysfunction [24]. In type II diabetes mice, genetic upregulation of SIRT1 [24] or treatment with resveratrol [25] consistently enhances insulin secretion and improves glucose tolerance. These data suggest that modulation of SIRT1 expression and/or activity would be of therapeutic interest against diabetes and diabetes-related cardiovascular complications.

SIRT1 also has beneficial effects on endothelial cells. In rat aorta, inhibition of SIRT1 ex vivo causes an impaired endothelium-dependent and nitric oxide (NO)mediated relaxation. Restoration of SIRT1 increases endothelial nitric oxide synthase (eNOS) expression by deacetylation of both lysine 496 and 506, indicating that SIRT1 directly regulates eNOS [26]. In apo $\mathrm{E}^{-/-}$mice fed on a high-fat diet, mice overexpressing endothelial cellspecific SIRT1 display blunted atherosclerosis due to an enhanced eNOS expression and preserved endotheliumdependent relaxation of the aorta, indicating a beneficial effect of SIRT1 as well as eNOS on atherogenesis [27]. Furthermore, NO donor increases SIRT1 expression and delays cell senescence in human endothelial cells. Calorie restriction fails to enhance SIRT1 expression in $\mathrm{eNOS}^{-/-}$mice [28], suggesting that NO may also directly modulate SIRT1 expression. This result confirms a protective role of SIRT1 and eNOS for the development of atherosclerosis.

In transgenic mice with heart-specific overexpression of SIRT1, beneficial effects are observed only in animals with a slight or moderate upregulation of SIRT1 mediated by an increased catalase expression through the FoxOdependent signaling pathway. Furthermore, heart-specific overexpression of SIRT1 retards aging of the heart and protects the heart from paraquat-induced oxidative stress [29].

\section{Mammalian Longevity Assurance Gene $\mathrm{p} 66^{\text {Shc }}$}

Shc protein is considered to be a regulator of longevity in mammals [30]. The mammalian Shc adaptor protein has three isoforms of 46,52 and $66 \mathrm{kDa}\left(\mathrm{p} 46^{\mathrm{Shc}}, \mathrm{p} 52^{\mathrm{Shc}}\right.$, and $\left.\mathrm{p} 66^{\mathrm{Shc}}\right)$. $\mathrm{p} 66^{\mathrm{Shc}}$ is the only isoform to play an important role as a redox enzyme implicated in mitochondrial ROS generation and translation of oxidative signals into apoptosis. Genetic deletion of $\mathrm{p} 66^{\text {Shc }}$ in the mouse leads to an extended life span which is correlated with a reduced production of intracellular oxidants and a decreased level of mtDNA content $[9,30] . \mathrm{p} 66^{\text {Shc }}$ predominantly localizes in the cytoplasm, with a small fraction $(10-40 \%)$ in the mitochondria. Ultraviolet light irradiation as well as oxidants such as $\mathrm{H}_{2} \mathrm{O}_{2}$ phosphorylate p $66^{\text {Shc }}$ on serine 36 , which in turn induces translocation of $\mathrm{p} 66^{\text {Shc }}$ to the mitochondria [31]. Activated $\mathrm{p} 66^{\text {Shc }}$ increases mitochondrial $\mathrm{Ca}^{2+}$ sensitivity and thus interacts with reduced cytochrome $\mathrm{c}$ to increase the production of $\mathrm{H}_{2} \mathrm{O}_{2}$ which promotes the opening of the mitochondrial permeability transition pore and leads to apoptosis [31]. p $66^{\text {Shc }}$ is also involved in $\mathrm{H}_{2} \mathrm{O}_{2}$-induced forkhead inhibition by increasing the phosphorylation of forkhead (Drosophila) homolog (rhabdomyosarcoma) like 1 (FKHRL1, also known as FoxO3a) [32]. Furthermore, according to cell type and stimulation, this ROS-sensitive protein plays an important role in regulating apoptosis by both p53-dependent and p53-independent mechanisms. In light of its pivotal role in ROS generation, many efforts have been made to investigate the pathophysiological role of $\mathrm{p} 66^{\text {Shc }}$ in aging and aging-related ROS-mediated diseases (fig. 1) [33].

It is reported that $\mathrm{p} 66^{\mathrm{Shc}}$ is highly expressed in fibroblasts from centenarians and in peripheral blood monocytes from diabetic patients. In line with the above, it is widely accepted that $\mathrm{p} 66^{\mathrm{Shc}}$ is linked to aging and/or ROSrelated diseases in humans. Genetic deletion is a useful tool to study the role of $\mathrm{p} 66^{\text {Shc }}$ protein under pathophysiological conditions since identical Shc genomic organization, transcript assembly and a high degree of amino acid identity have been reported in mice and humans [33].

In line with this hypothesis, $\mathrm{p} 66^{\text {Shc-/- }}$ mice are protected from aging-dependent endothelial dysfunction [34]. Wild-type mice display aging-dependent loss of $\mathrm{NO}$-mediated vasorelaxation, while $\mathrm{p} 66^{\text {Shc-l- }}$ mice show a maintained ability to relax by virtue of an increased $\mathrm{NO}$ bioavailability, lower aortic $\mathrm{O}_{2}^{-}$levels and reduced aortic 3-nitrotyrosine content (fig. 2). In endothelial cell culture experiments, inhibition of $\mathrm{p} 66^{\mathrm{Shc}}$ increases NO production by phosphorylating eNOS on serine 1177 [35]. There- 


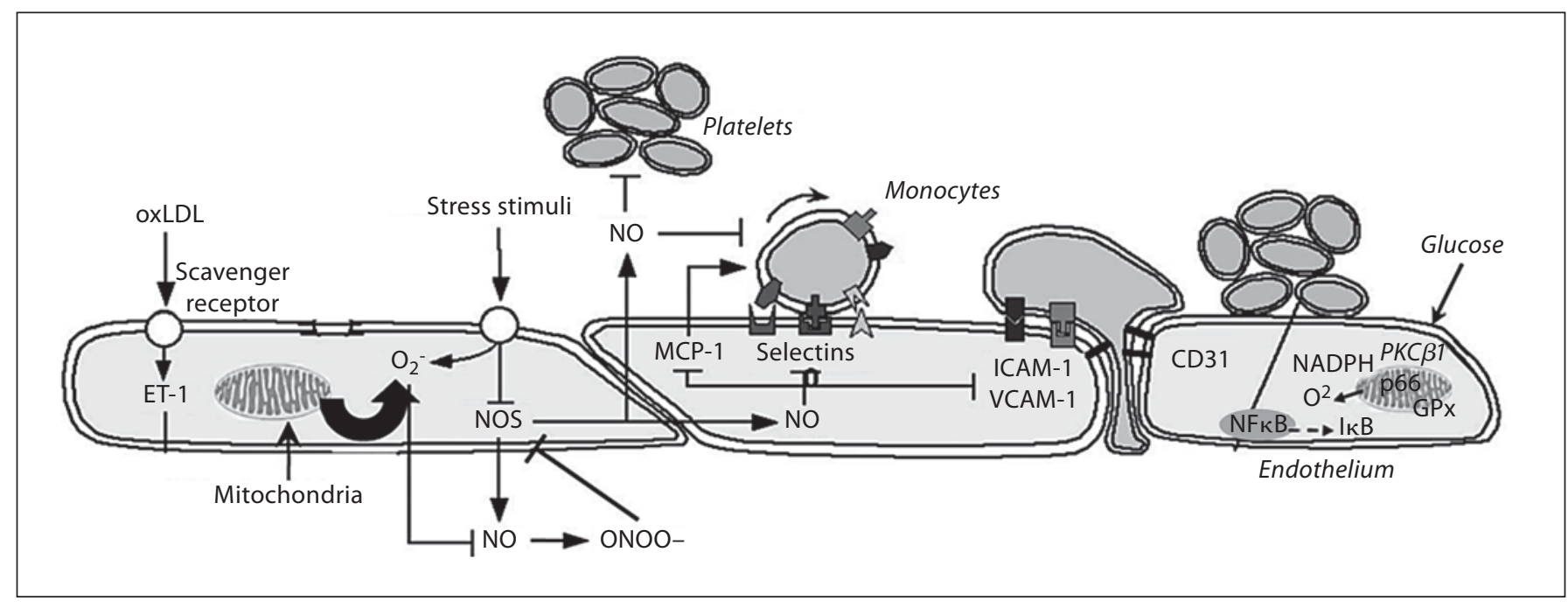

Fig. 1. Schematic representation of the endothelium and some of its key derived vasoactive substances. $\mathrm{p} 66^{\text {Shc }}$ is principally located in the mitochondria, where it contributes to the production of superoxide anion $\left(\mathrm{O}_{2}^{-}\right)$, radicals which scavenge NO to form peroxynitrite $\left(\mathrm{ONOO}^{-}\right)$. Different stress stimuli (e.g. glucose, oxLDL, hypertension, shear stress) increase in number and occurrence with age and enhance the production of $\mathrm{O}_{2}^{-}$, thus exacerbating endothelial dysfunction. oxLDL $=$ Oxidized low-density lipopro- tein; ET-1 = endothelin-1; NOS = NO synthase; $\mathrm{ONOO}=$ peroxynitrite; $\mathrm{MCP}-1$ = monocyte chemotactic protein-1; ICAM = intercellular adhesion molecule; VCAM = vascular cells adhesion

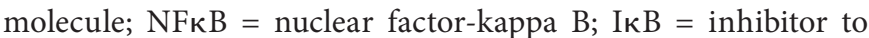
$\mathrm{NF \kappa B}$; NADPH = nicotinamide adenine dinucleotide phosphateoxidase $\mathrm{PKC1}=$ protein kinase $\mathrm{C}-1 ; \mathrm{GPx}=$ glutathione peroxidase [33].

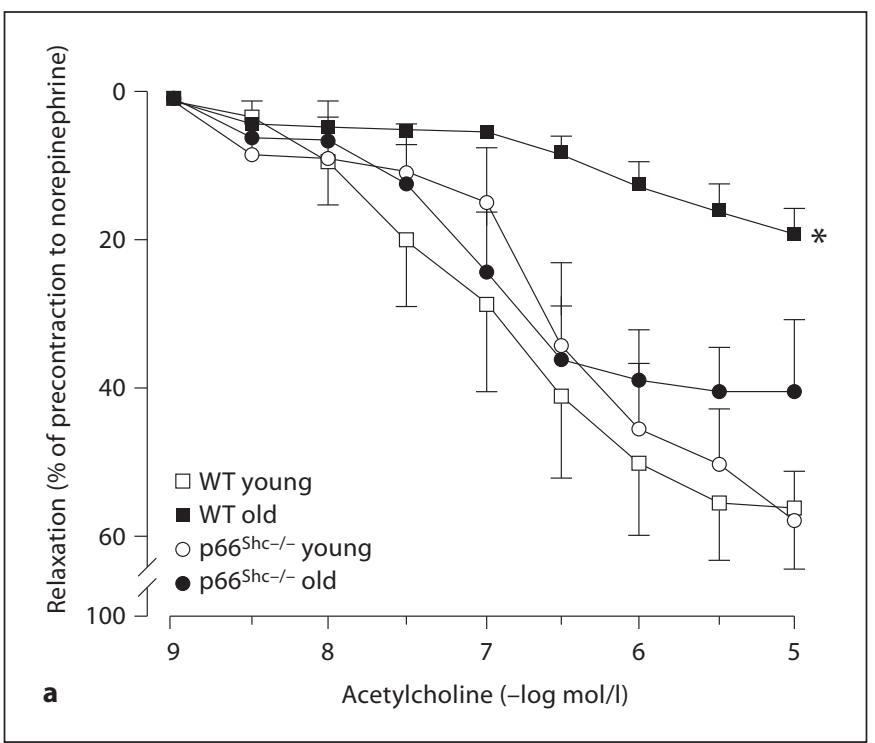

Fig. 2. a Age-dependent changes in endothelium-dependent relaxation of wild-type (WT) and p $66^{\text {Shc-l- }}$ aortas. Line graphs show concentration-response curves to acetylcholine. Results are presented as means \pm SEM; $n=7$ in each group. $\mathbf{b}$ Bar graph showing

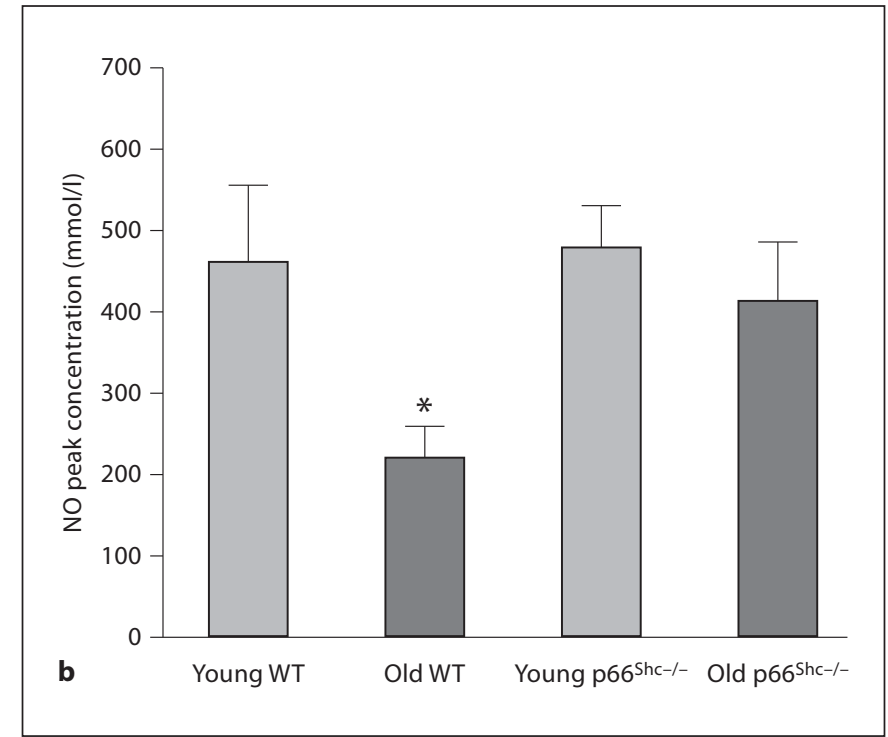

peak concentrations of NO in young (gray bars) and old (black bars) WT and $\mathrm{p} 66^{\mathrm{Shc}-1-}$ mice, respectively. Results are presented as means \pm SEM; $\mathrm{n}=4$ to 6 in each group. ${ }^{*} \mathrm{p}<0.05$ vs. young WT [34]. 


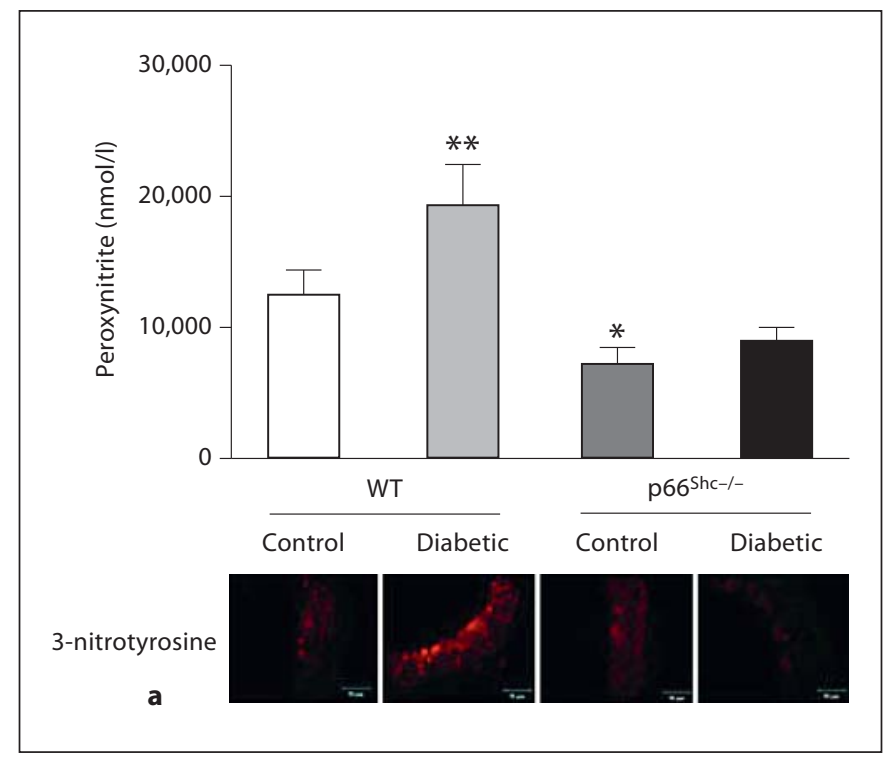

Fig. 3. a Bar graphs show $\mathrm{ONOO}^{-}$levels determined by chemiluminescence. Results are presented as means \pm SEM; $n=6$ in each group. Immunostaining of nitrotyrosine residues in aortas from control and diabetic WT and p66 ${ }^{\text {Shc- }- \text { - }}$ mice is shown. ${ }^{*} \mathrm{p}<0.05$ for $\mathrm{p} 66^{\text {Shc-l- }}$ control vs. WT control; ${ }^{* *} \mathrm{p}<0.05$ for WT diabetic

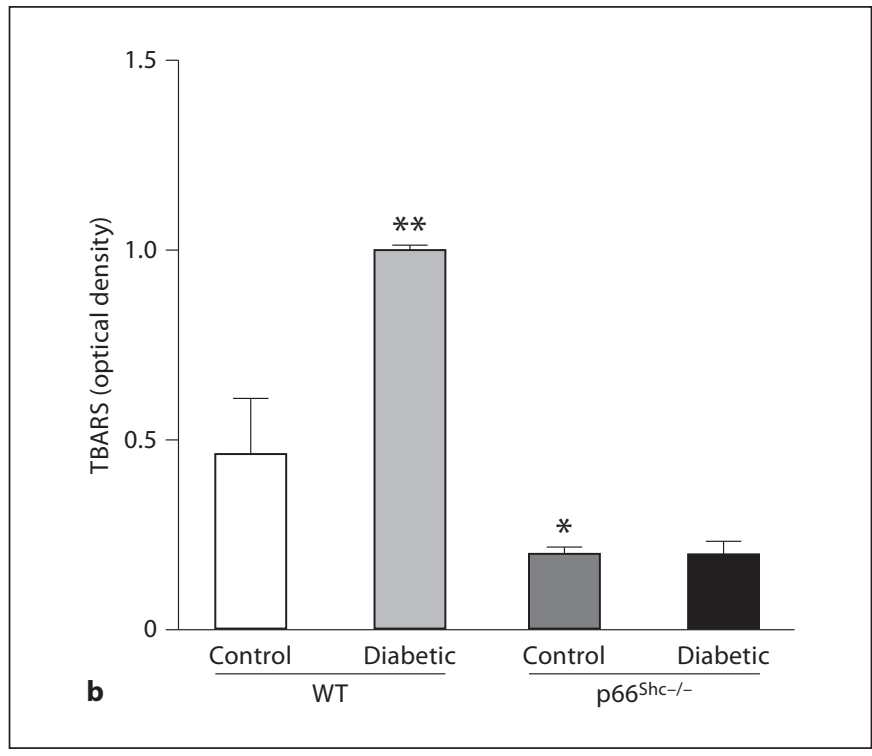

vs. WT control. b TBARS levels. Bar graphs show TBARS levels in aortas from control and diabetic WT and $\mathrm{p} 66^{\text {Shc-l- }}$ mice. Results are presented as means \pm SEM; $\mathrm{n}=4$ in each group. ${ }^{*} \mathrm{p}<0.05$ for $\mathrm{p} 66^{\text {Shc- }- \text { - }}$ control vs. WT control; ${ }^{* *} \mathrm{p}<0.05$ for WT diabetic vs. WT control [37].

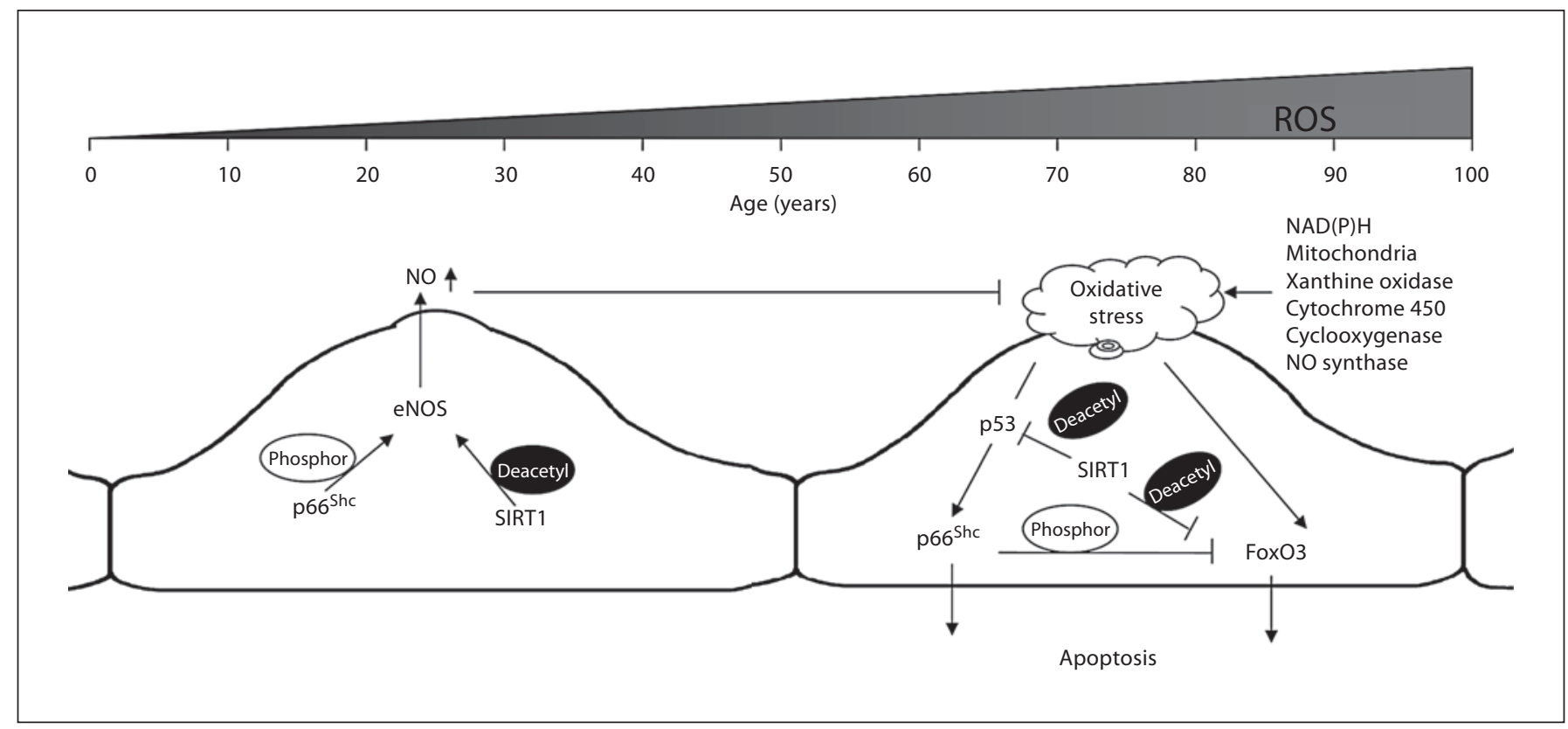

Fig. 4. Schematic representation of effects of $p 66^{\text {Shc }}$ and SIRT1 in anti-aging treatments. The production of ROS increase with aging. p $66^{\text {Shc }}$ activates eNOS by phosphorylation while SIRT1 maintains eNOS protein by deacetylation. ROS-induced apoptosis goes through FoxO3 or the p53 pathway. Oxidative stress acti- vates FoxO3 by a combination of acetylation and phosphorylation events. p6 $6{ }^{\text {Shc }}$ mediates in $\mathrm{H}_{2} \mathrm{O}_{2}$-induced phosphorylation, whereas SIRT1 directly deacetylates effect on FoxO3. p $66^{\mathrm{Shc}}$ is a downstreaming transcriptional target of p53, while SIRT1 negatively regulates the ability of $\mathrm{p} 53$ by protein deacetylation. 
fore, $\mathrm{p} 66^{\text {Shc }}$ adaptor protein is regarded as a key player in aging-dependent loss of endothelial integrity and NO bioavailability.

Furthermore, $\mathrm{p} 6^{\text {Shc- } /-}$ apoE ${ }^{-/-}$mice fed with a highfat diet display lower levels of lipid peroxidation compared to control mice, thus suggesting a possible role of p $66^{\text {Shc }}$ in the oxidative modification of lipoprotein. Compared with control littermates fed high-fat diets, $\mathrm{p} 66^{\mathrm{Shc}-I-}$ apoE ${ }^{-/-}$mice are shown to have less aortic lesion formation accompanied by a decrease in intimal foam cell formation and lower levels of systemic plasma isoprostanes [36]. Additional studies confirm a tight link between plasma levels of LDL-cholesterol and $\mathrm{p} 66^{\text {Shc }}$ in pacemaker-implanted patients, thus underscoring that $\mathrm{p} 66^{\mathrm{Shc}}$ plays a role in translating the effects of different agingrelated risk factors into arterial dysfunction and early atherosclerotic lesion [33].

High glucose induces a cascade of cellular events which increase the production of free radicals, thus decreasing NO bioavailability and eventually leading to vascular dysfunction. In conditions of raised glucose plasma levels, $\mathrm{p} 66^{\text {Shc }}$ is known to oxidize cytochrome c and, in turn, generate proapoptotic ROS through a protein kinase $c-\beta$ dependent pathway [31]. In line with the above, $\mathrm{p} 66^{\mathrm{Shc}}$ protein expression is increased in aortas of type I diabetes mice [37]. p66 ${ }^{\text {Shc-l- }}$ mice have comparable glucose levels as control mice [37, 38], indicating that knocking out $\mathrm{p} 66^{\text {shc }}$ protein does not alter glycemic levels. However, they are protected from diabetic glomerulopathy as demonstrated by reduced glomerular cell apoptosis and decreased extracellular matrix deposition, indicating the pivotal role of $\mathrm{p} 66^{\mathrm{Shc}}$ in translating ROSmediated insults into apoptosis [38]. Furthermore, streptozotocin-induced diabetic $\mathrm{p} 66^{\mathrm{Shc}-/-}$ mice are protected against endothelial dysfunction via a blunted ROS production and the consequently preserved $\mathrm{NO}$ bioavailability (fig. 3). This finding reinforces the concept of a causal relationship between high glucose, ROS, $\mathrm{p} 66^{\mathrm{Shc}}$ and vascular dysfunction [37].

Notably, a definite balance between ROS damage and endothelial progenitor cell (EPC)-mediated repair exists and guarantees endothelial integrity. In keeping with this theory, the increased ROS production observed in aged mice or mice with a pathophysiological condition, such as hyperglycemia, could explain both impairment of endothelial function and loss of EPC-mediated repair, which ultimately leads to vascular dysfunction [39]. These changes, however, are not observed in $\mathrm{p} 66^{\text {Shc }}$ knockout mice which present lower levels of ROS and a preserved endothelial function. Therefore, the mitochon- drial adaptor protein $\mathrm{p} 66^{\mathrm{Shc}}$ represents a promising target protein for slowing the process of aging and blunting aging-related ROS generation [33].

\section{Clinical Perspectives and Conclusions}

The signaling pathways relevant to aging discussed here are currently under intensive investigation as potential targets for new anti-aging treatments based either on reducing oxidative damage or enhancing cell defenses. Indeed, p66 ${ }^{\text {Shc }}$ and SIRT1 share multiple molecular targets (fig. 4). First, both proteins enhance NO bioavailability through eNOS upregulation/activation and/or reduction of NO scavenging. Indeed, $\mathrm{p} 66^{\text {Shc }}$ activates eNOS by phosphorylating serine 1177 [35], while SIRT1 regulates eNOS activity by deacetylation on both lysine 496 and 506 [26]. Second, upregulation of FoxO3, which is important for increasing activity of antioxidant systems such as catalase and superoxide dismutases, is mediated by both p $66^{\text {Shc }}$ and SIRT1 through phosphorylation and acetylation, respectively. In fact, p66Shc plays a role in $\mathrm{H}_{2} \mathrm{O}_{2}$ induced phosphorylation of FoxO3 [32], whereas SIRT1 has a direct effect on deacetylation of FoxO3 [20]. Third, p66Shc is a downstream target of p53 [30], whereas SIRT1 negatively regulates p53 by protein deacetylation [24].

It may seem optimistic to assume that simply downregulating $\mathrm{p} 66^{\text {Shc }}$, upregulating SIRT1, or a combination of the two will effectively slow down aging in humans and shift the pathophysiological imbalance towards a normalized condition. SIRT1 exerts multiple protective effects. Nevertheless, physical training is reported to restore SIRT1 protein in both heart and adipose tissue of aged mice, supporting previous evidence showing beneficial effects of exercise training [40]. Furthermore, resveratrol, one of the polyphenolic compounds in grapes and wine, is reported to be a small-molecule activator of SIRT1 $[21,25]$, which may be worth investigating as a potential treatment for aging-related human diseases. $p 66^{\text {Shc }}$ is a mitochondrial protein mediating oxidative stress $[9$, 33]. Genetic deletion of p66Shc protein has shown multiple beneficial effects in the process of atherosclerosis and diabetes in experimental animal models. Therefore, it is believed that the inhibition of p66Shc is the more attractive therapeutic option to reduce oxidative stress in human rather than antioxidant treatment as previously discussed [33]. The target proteins described in this review may offer new insights to better characterize underlying mechanisms of aging and may potentially represent novel therapeutic targets to prevent aging-related disease. 


\section{References}

$\checkmark 1$ Sniderman AD, Furberg CD: Age as a modifiable risk factor for cardiovascular disease. Lancet 2008;371:1547-1549.

2 Dhahbi JM, et al: Temporal linkage between the phenotypic and genomic responses to caloric restriction. Proc Natl Acad Sci USA 2004;101:5524-5529.

- 3 McCay CM, Crowell MF, Maynard LA: The effect of retarded growth upon the length of life span and upon the ultimate body size. 1935. Nutrition 1989;5:155-172.

4 Colman RJ, et al: Caloric restriction delays disease onset and mortality in rhesus monkeys. Science 2009;325:201-204.

5 DeLany JP, et al: Long-term calorie restriction reduces energy expenditure in aging monkeys. J Gerontol A Biol Sci Med Sci 1999; 54:B5-B11, discussion B12-B13.

-6 Fontana L, et al: Long-term calorie restriction is highly effective in reducing the risk for atherosclerosis in humans. Proc Natl Acad Sci USA 2004;101:6659-6663.

7 Martinez-Gonzalez MA, et al: Mediterranean food pattern and the primary prevention of chronic disease: recent developments. Nutr Rev 2009;67(suppl 1):S111-S116.

$\checkmark 8$ Harman D: Aging: a theory based on free radical and radiation chemistry. J Gerontol 1956;11:298-300.

9 Shi Y, Camici GG, Luscher TF: Cardiovascular determinants of life span. Pflügers Arch 2010;459:315-324.

10 Paradies G, et al: Role of cardiolipin peroxidation and $\mathrm{Ca} 2+$ in mitochondrial dysfunction and disease. Cell Calcium 2009;45:643650.

11 Barcelo F, et al: Mediterranean-style diet effect on the structural properties of the erythrocyte cell membrane of hypertensive patients: the Prevencion con Dieta Mediterranea Study. Hypertension 2009;54:11431150.

12 Lass A, et al: Caloric restriction prevents ageassociated accrual of oxidative damage to mouse skeletal muscle mitochondria. Free Radic Biol Med 1998;25:1089-1097.

13 Shigenaga MK, Hagen TM, Ames BN: Oxidative damage and mitochondrial decay in aging. Proc Natl Acad Sci USA 1994;91: 10771-10778
14 Cassano P, et al: Measurement of the 4,834bp mitochondrial DNA deletion level in aging rat liver and brain subjected or not to caloric restriction diet. Ann NY Acad Sci 2004; 1019:269-273.

15 Hao J, et al: Hydroxytyrosol promotes mitochondrial biogenesis and mitochondrial function in 3T3-L1 adipocytes. J Nutr Biochem 2009, Epub ahead of print.

16 Pamplona R, Barja G, Portero-Otin M: Membrane fatty acid unsaturation, protection against oxidative stress, and maximum life span: a homeoviscous-longevity adaptation? Ann NY Acad Sci 2002;959:475-490.

17 Feuers RJ: The effects of dietary restriction on mitochondrial dysfunction in aging. Ann NY Acad Sci 1998;854:192-201.

18 Rao G, et al: Effect of dietary restriction on the age-dependent changes in the expression of antioxidant enzymes in rat liver. J Nutr 1990;120:602-609.

19 Luhtala TA, et al: Dietary restriction attenuates age-related increases in rat skeletal muscle antioxidant enzyme activities. J Gerontol 1994;49:B231-B238.

20 Brunet A, et al: Stress-dependent regulation of FOXO transcription factors by the SIRT1 deacetylase. Science 2004;303:2011-2015.

$21 \mathrm{Yu}$ J, Auwerx J: The role of sirtuins in the control of metabolic homeostasis. Ann NY Acad Sci 2009;1173(suppl 1):E10-E19.

-22 Rodgers JT, et al: Nutrient control of glucose homeostasis through a complex of PGC-1alpha and SIRT1. Nature 2005;434:113-118.

23 Moynihan KA, et al: Increased dosage of mammalian Sir2 in pancreatic beta cells enhances glucose-stimulated insulin secretion in mice. Cell Metab 2005;2:105-117.

24 Orimo M, et al: Protective role of SIRT1 in diabetic vascular dysfunction. Arterioscler Thromb Vasc Biol 2009;29:889-894.

25 Milne JC, et al: Small molecule activators of SIRT1 as therapeutics for the treatment of type 2 diabetes. Nature 2007;450:712-716.

26 Mattagajasingh I, et al: SIRT1 promotes endothelium-dependent vascular relaxation by activating endothelial nitric oxide synthase. Proc Natl Acad Sci USA 2007;104:1485514860.

27 Zhang QJ, et al: Endothelium-specific overexpression of class III deacetylase SIRT1 decreases atherosclerosis in apolipoprotein Edeficient mice. Cardiovasc Res 2008;80: 191-199.
28 Nisoli E, et al: Calorie restriction promotes mitochondrial biogenesis by inducing the expression of eNOS. Science 2005;310:314317.

29 Alcendor RR, et al: Sirt1 regulates aging and resistance to oxidative stress in the heart. Circ Res 2007;100:1512-1521

30 Migliaccio E, et al: The p66shc adaptor protein controls oxidative stress response and life span in mammals. Nature 1999;402:309313.

31 Pinton $\mathrm{P}$, et al: Protein kinase $\mathrm{C}$ beta and prolyl isomerase 1 regulate mitochondrial effects of the life-span determinant p66Shc. Science 2007;315:659-663.

32 Nemoto S, Finkel T: Redox regulation of forkhead proteins through a p66shc-dependent signaling pathway. Science 2002;295: 2450-2452.

33 Camici GG, et al: The role of p66Shc deletion in age-associated arterial dysfunction and disease states. J Appl Physiol 2008; 105:16281631.

34 Francia P, et al: Deletion of p66shc gene protects against age-related endothelial dysfunction. Circulation 2004;110:2889-2895.

35 Yamamori T, et al: P66shc regulates endothelial NO production and endotheliumdependent vasorelaxation: implications for age-associated vascular dysfunction. J Mol Cell Cardiol 2005;39:992-995.

36 Napoli C, et al: Deletion of the p66Shc longevity gene reduces systemic and tissue oxidative stress, vascular cell apoptosis, and early atherogenesis in mice fed a high-fat diet. Proc Natl Acad Sci USA 2003;100:21122116.

37 Camici GG, et al: Genetic deletion of p66(Shc) adaptor protein prevents hyperglycemia-induced endothelial dysfunction and oxidative stress. Proc Natl Acad Sci USA 2007;104:5217-5222.

38 Menini S, et al: Deletion of p66Shc longevity gene protects against experimental diabetic glomerulopathy by preventing diabetes-induced oxidative stress. Diabetes 2006;55: $1642-1650$

39 Di Stefano V, et al: p66ShcA modulates oxidative stress and survival of endothelial progenitor cells in response to high glucose. Cardiovasc Res 2009;82:421-429.

40 Ferrara N, et al: Exercise training promotes SIRT1 activity in aged rats. Rejuvenation Res 2008;11:139-150. 